\title{
Toward Top-Down Determination of PEGylation Site Using MALDI In-Source Decay MS Analysis
}

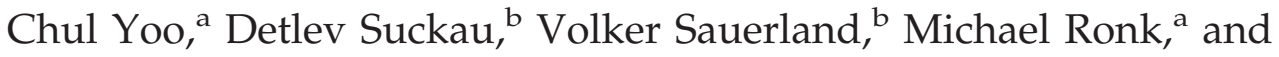 \\ Minhui $\mathrm{Ma}^{\mathrm{a}}$ \\ a Analytical Research and Development, Amgen Inc., Thousand Oaks, California, USA \\ ${ }^{\mathrm{b}}$ Bruker Daltonik GmbH, Bremen, Germany
}

A novel matrix assisted laser desorption/ionization (MALDI)-based mass spectrometric approach has been evaluated to rapidly analyze a custom designed PEGylated peptide that is 31 residues long and conjugated with $20 \mathrm{kDa}$ linear polyethylene glycol (PEG) at the side chain of Lys. MALDI-TOF MS provided sufficiently high resolution to allow observation of each of

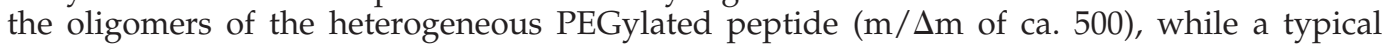
ESI-MS spectrum of this molecule was extremely complex and unresolved. Reflector in-source decay (reISD) analysis using MALDI-TOF MS was attempted to identify the PEGylation site at intact molecular level without any sample treatment. An reISD spectrum of the free peptide was observed with abundant c-, y-, and [z +2$]$-fragment ion series, whereas, in the fragmented PEGylated peptide, the fragment ion series were truncated at the residue where PEG was attached. Therefore, a direct comparison of these top-down reISD spectra suggested the location of the PEGylation site. Results from this study demonstrate a clear analytical utility of the ISD technique to characterize structural aspects of heterogeneous biomolecules. (J Am Soc Mass Spectrom 2009, 20, 326-333) @ 2009 American Society for Mass Spectrometry

A significant challenge is associated with development of biomolecules into effective therapeutic use due to their limited chemical and physical stability. In particular, short plasma half-life of biotherapeutics may lead to poor efficacy in humans, and, therefore, developing methods to achieve longer circulation times at low dosage volumes is required. To obtain such improved stability, biomolecules can be chemically modified. One of the most successful approaches currently employed is to covalently attach inert, nontoxic, and nonantigenic polymers, such as polyethylene glycol (PEG), to active molecules, a strategy termed PEGylation [1-6]. Due to its favorable properties, PEG is approved by FDA [5] for oral, injection, and dermal administration to humans. PEGylation technology has been successfully developed and applied to achieve prolonged in vivo circulation of biomolecules in the body for effective potency, as demonstrated by a series of marketed PEGylated therapeutics, including Neulasta ${ }^{\circ}$, or pegfilgrastim $[5,7,8]$.

Typically, the conjugation of PEG of ca. $20 \mathrm{kDa}$ or larger to biotherapeutics has proven efficient by providing desired stability $[4,9,10]$. However, tremendous analytical challenges are associated with studying such heterogeneous molecules [11], where confident deter-

Address reprint requests to Dr. C. Yoo, Analytical Research and Development, Amgen, Inc., One Amgen Center Drive, Mail stop 25-2-A, Thousand Oaks, CA 91320, USA. E-mail: cyoo@amgen.com mination of the PEGylation site is one of the important requirements to fully characterize and understand PEGylated molecules. Previously, several groups have reported on the identification of the site at which PEG was conjugated [12], where PEG of relatively small size were commonly used [13-15]. Due to a lower degree of polymerization associated with smaller PEG, an analysis was readily performed in these cases. Also, these studies mostly involved enzymatic digestions to selectively analyze the smaller fragments bearing potential PEGylation sites by mass mapping. Although this bottom-up approach may provide useful information, it has a disadvantage of resulting in excessive numbers of peptides to complicate analysis. This issue becomes more serious when the biomolecules to be PEGylated are of large size, as it is usually the case for many biotherapeutics, and contain multiple potential conjugation sites. Also, from practical point of view, this procedure involves lengthy sample preparations that are prone to sample loss, resulting in reduced sensitivity. Therefore, it is most ideal to be able to study these types of molecules in their intact forms without any chemical or enzymatic modifications.

Matrix assisted laser desorption/ionization mass spectrometry (MALDI-MS) has been widely accepted as a useful means to study various types of biomolecules due to its soft ionization properties [16, 17]. Unlike electrospray ionization (ESI) that produces multiply charged ions, the MALDI process almost exclusively 
generates singly charged ions [18], which can greatly help reduce complexity of mass spectra for easier and more confident data interpretation. MALDI is most conveniently combined with a time-of-flight (TOF) mass analyzer that can provide sufficiently highresolution and mass accuracy in a wide achievable mass range to resolve large heterogeneous molecules [19]. Due to its remarkable sensitivity, low sample consumption, ease of sample preparation, and high-throughput capability, MALDI-TOF MS has also been successfully used for the analysis of synthetic polymers [20-23]. Further advancement has led to the development of MALDI-TOF/TOF MS [24-26], which has been extensively used in proteomics applications for characterizing biomarkers and important post-translational modifications (PTMs) [27-30]. In addition, its reflector in-source decay (reISD) capability enabled the topdown analysis of intact proteins.

In-source decay (ISD), a unique fragmentation technique available with MALDI-MS [31-35], is a fast fragmentation process occurring at the nanosecond time scale inside the source region, rapidly following the laser shot [36]. According to Takayama's model, the formation of the specific $\mathrm{c}$ and $\mathrm{z}$ ions in ISD spectra arises from hydrogen radical transfer from matrix to analyte [37-39], inducing fragmentation at the position where the transfer occurs. Therefore, ISD fragmentation does not exhibit any preference for labile bonds but occurs uniformly across the protein sequence and can be advantageous by generating relatively simple fragment ion spectra even from large proteins. Previously, ISD has been shown to be useful in determining primary structure of biomolecules [35]. ISD tends to work very efficiently for analyzing large molecules, as recently demonstrated in the study of intact proteins and its applications also included the characterizations of PTMs, such as disulfide bonds and phosphorylation $[25,31,36]$. Based on the unique mechanism by which ISD occurs and the fact that modifications usually remain intact during this fragmentation process for the purpose of identification and localization [31], it appears that ISD can be useful to study purposely modified biomolecules, such as PEGylated peptides, at the intact molecular level. In this work, we present preliminary findings obtained for the determination of a PEGylation site by ISD analysis using a custom constructed PEGylated peptide as a model compound without any chemical or enzymatic treatments.

\section{Experimental}

\section{Materials}

An in-house synthesized peptide that consists of 31 amino acids has been purified and conjugated to polyethylene glycol at Amgen Inc. (Thousand Oaks, CA). As previously described [40], both termini of this peptide remain blocked by acetylation at the $\mathrm{N}$-terminus and $-\mathrm{NH}_{2}$ at the C-terminus. The sequence consists of the following: Ac-WVTHR ${ }^{*}$ LAGLLSR $*$ SGGVVK* $K^{19}$ NFVPTDVGPXAF-NH $\mathrm{N}_{2}$, where $20 \mathrm{kDa}$ linear mPEGaldehyde (Sunbright; NOF Corp., Tokyo, Japan) was conjugated at the $\mathrm{Lys}^{19}$ residue through the $\varepsilon$-amino group. $\mathrm{R}^{*}, \mathrm{~K}^{*}$, and $\mathrm{X}$ represent non-natural amino acids incorporated for citrulline, homoArg, and naphthylAla, respectively.

Trifluoroacetic acid (TFA) was obtained from J. T. Baker (Phillipsburg, NJ), formic acid from Riedel-de Haen (Morristown, NJ), and acetonitrile from Burdick and Jackson (Morristown, NJ). DI water was purified using Milli-Q system (18.2 $\mathrm{M} \Omega \cdot \mathrm{cm}$; Millipore, Billerica, MA). Sinapinic acid, 2,5-dihydroxybenzoic acid (DHB), and the MALDI peptide calibration standard were obtained from Bruker Daltonics (Bremen, Germany) and used without further purifications. A PEG standard of $22 \mathrm{kDa}$ used for the MALDI analysis was from Polymer Standards Service (Mainz, Germany). Methanol and ethanol were purchased from Sigma-Aldrich (Deissenhofen, Germany).

\section{ESI-LTQ Orbitrap MS Analysis}

A small particle-packed reversed-phase HPLC column (Zorbax SB-C18, $2.1 \times 100$ mm, $1.8 \mu \mathrm{m}$; Agilent Technologies, Palo Alto, CA) was used to obtain highresolution separation. Chromatographic analysis was performed using an Agilent $1100 \mathrm{HPLC}$ system operated at a flow rate of $0.2 \mathrm{~mL} / \mathrm{min}$ with a column temperature controlled at $40{ }^{\circ} \mathrm{C}$. The mobile phase was comprised of $0.05 \%$ TFA and $0.05 \%$ formic acid in $100 \%$ DI water for $\mathrm{A}$ and in $100 \%$ acetonitrile for B. The following gradient profile was used: $10 \%$ to $30 \%$ B in 10 $\min , 30 \%$ to $55 \%$ B in $35 \mathrm{~min}, 55 \%$ to $100 \%$ B in $2 \mathrm{~min}$, hold at $100 \%$ B for $1 \mathrm{~min}, 100 \%$ to $10 \% \mathrm{~B}$ in $1 \mathrm{~min}$, and hold at $10 \%$ B for $16 \mathrm{~min}$. Approximately $5 \mu \mathrm{g}$ of the PEGylated peptide was loaded onto the column. UV detection was monitored at $215 \mathrm{~nm}$. The HPLC was interfaced directly to an LTQ Orbitrap MS equipped with an ESI source (Thermo, San Jose, CA) for the acquisition of mass spectra using Xcalibur software (version 2.0 SR1; Thermo). The $20 \mathrm{kDa}$ PEG sample, prepared at a concentration of $1 \mu \mathrm{g} / \mathrm{mL}$ in DI water, was directly infused into the LTQ Orbitrap MS via ESI at a flow rate of $10 \mu \mathrm{L} / \mathrm{min}$. The LTQ Orbitrap is a hybrid high-resolution MS configured with a linear ion trap as a front end for setting MS parameters and an electrostatic orbital ion trap as a back end for the acquisition of mass spectra of high-resolution and high accuracy. Connecting the two components of the mass spectrometer is a curved linear trap, or C-trap, which is used to store and condition ions for introduction into the Orbitrap mass analyzer.

\section{MALDI-MS Analysis and Data Interpretation}

For the acquisition of the MALDI spectrum of the PEGylated peptide, a sinapinic acid "crystal seed" preparation method was used. From a saturated solution of 

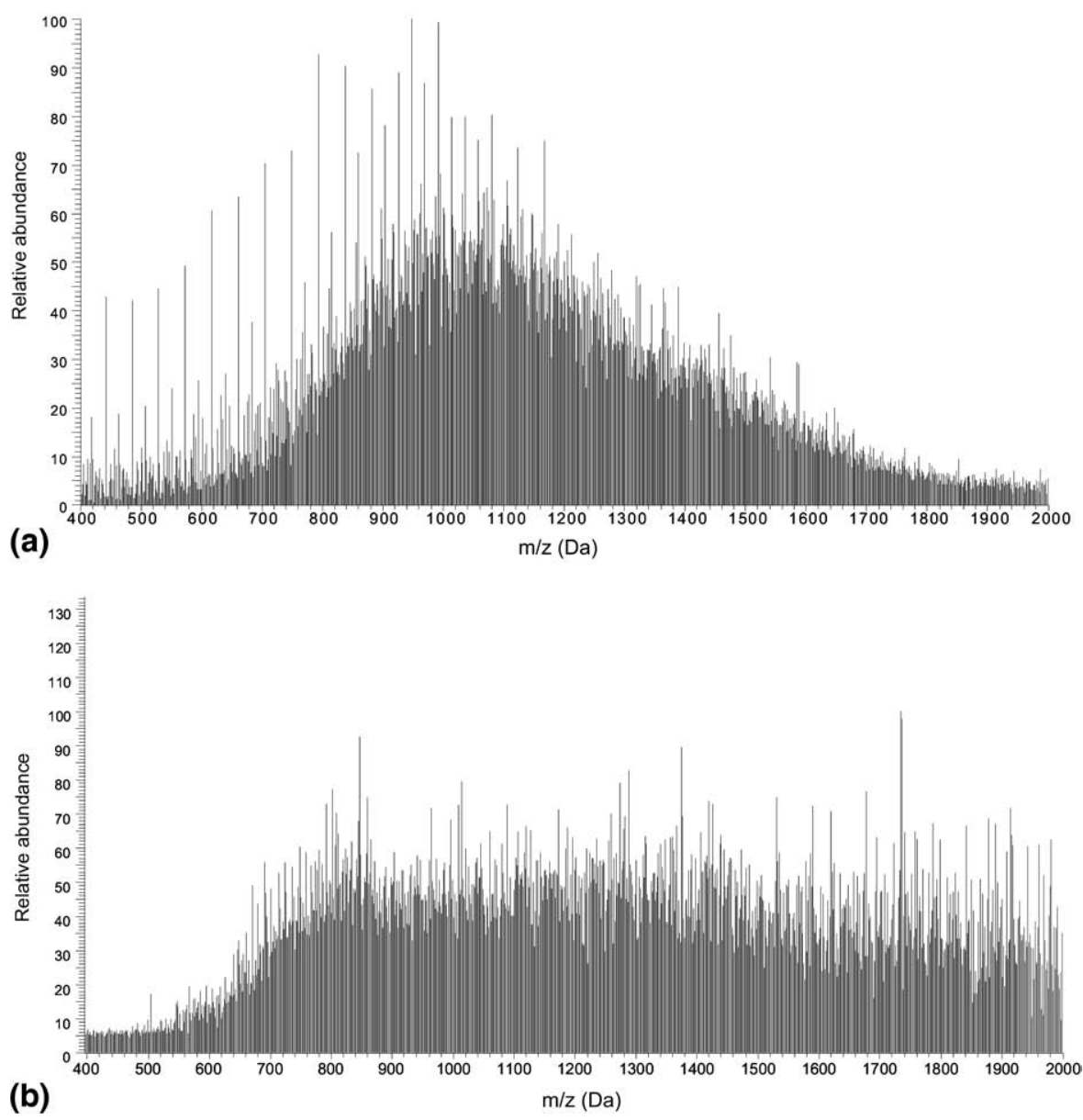

Figure 1. ESI-linear ion trap MS spectrum of (a) $20 \mathrm{kDa}$ linear PEG and (b) PEGylated peptide.

sinapinic acid prepared in ethanol, a thin-layer was formed on a stainless steel MALDI target plate. The PEGylated peptide was dissolved in 50\% methanol/ $50 \%$ water at a concentration of $1 \mathrm{mg} / \mathrm{mL}$ and mixed at
1:1 ratio with a saturated sinapinic acid containing $30 \%$ acetonitrile and $0.1 \%$ TFA; $1 \mu \mathrm{L}$ of this mixture was spotted onto the thin-layer. For the acquisition of the reISD spectra, a saturated solution of DHB prepared in

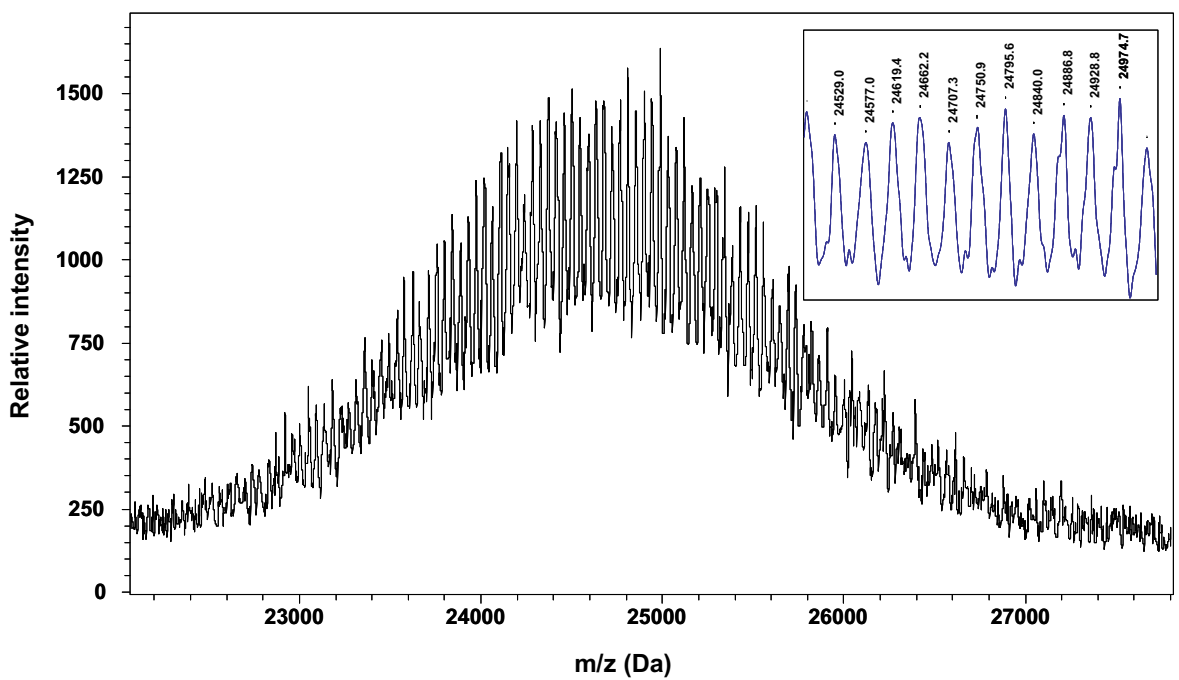

Figure 2. Linear MALDI-TOF MS spectrum obtained for the PEGylated peptide in which the ca. 3.5 kDa peptide is covalently attached to $20 \mathrm{kDa}$ linear PEG (inset: oligomer structure of the $[\mathrm{M}+\mathrm{H}]^{+}$ ions resolved. The inset differs by the level of smoothing applied to the spectra). 


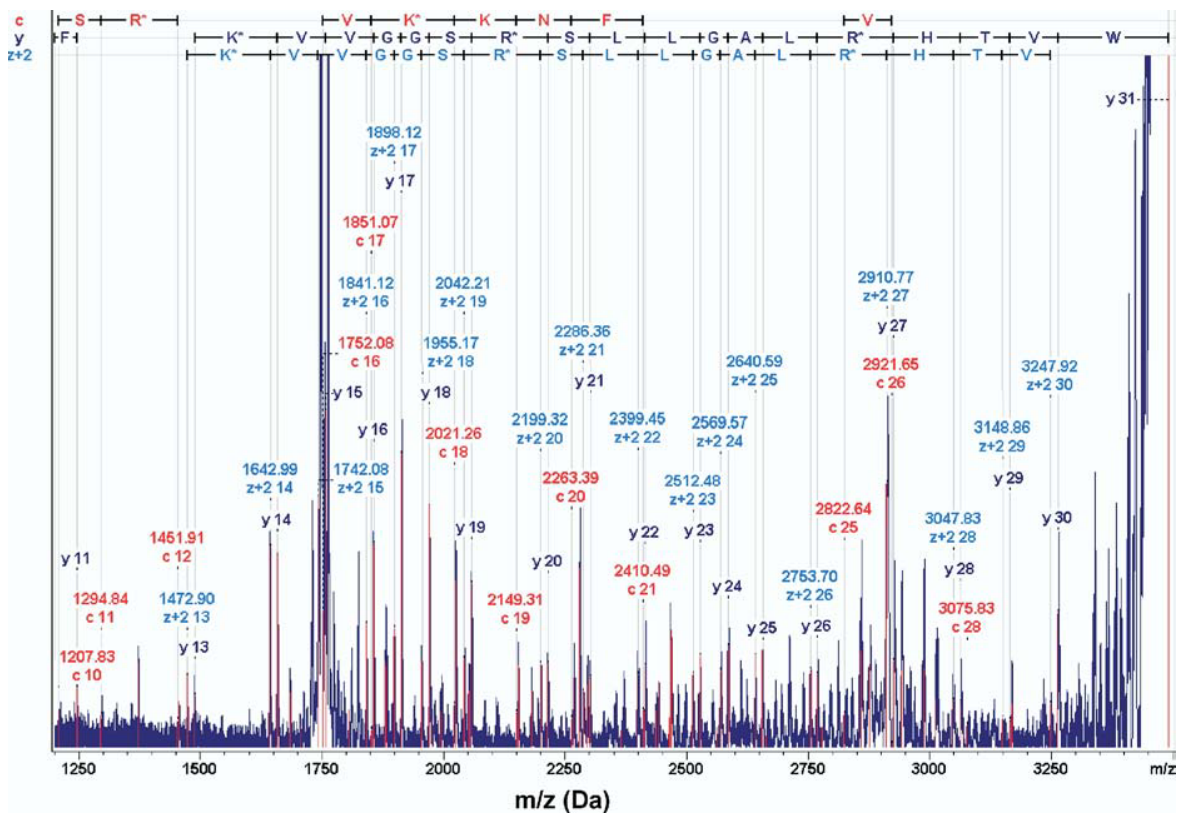

Figure 3. reISD MALDI MS spectrum obtained for the free peptide used for PEGylation ( $\mathrm{R}^{*}$ for citrulline; $\mathrm{K}^{*}$ for homoArg). Matching fragment ions are assigned.

$10 \%$ acetonitrile was used as a matrix. The free peptide of $500 \mathrm{fmol} / \mu \mathrm{L}$ and the PEGylated peptide prepared above were each mixed with DHB, where $2 \mu \mathrm{L}$ of each of these mixtures was spotted onto a target plate.

A Bruker Ultraflex III MALDI-TOF/TOF MS equipped with $200 \mathrm{~Hz}$ solid-state smartbeam laser [41], LIFT-TOF/TOF assembly, and gridless ion reflector [25] was used for all MALDI MS measurements. ReISD spectra were obtained as previously described [25, 33]. In general, increased laser fluence, delayed extraction, and the choice of matrix are critical for the formation of ISD fragment ions. Six thousand to 8000 single laser shots were acquired for each reISD spectrum. External calibration of the spectra was carried out using PEG of $22 \mathrm{kDa}$ for the analysis of intact PEGylated peptide and a peptide calibration standard for reISD fragmented PEGylated peptide. Spectra were acquired and processed using the Compass 1.2 software package (Bruker Daltonics). BioTools version 3.0 was used for the topdown sequencing analysis of the peptides.

\section{Results and Discussion}

\section{ESI-MS and MALDI-TOF MS Analysis of PEGylated Peptide}

In contrast to unmodified proteins, PEGylated biomolecules generally show oligomeric distribution of the polymer with mass differences of $44 \mathrm{Da}$ between the oligomers. Previously, MALDI-TOF MS has been shown to be useful in resolving the polymeric distributions of various polymers and PEGylated molecules conjugated with relatively small size PEG [14, 42-46], where singly charged ions are formed and detector saturation is not significant [23]. However, it is important to be able to analyze and resolve PEGylated biomolecules conjugated with PEG of large size, i.e., 20 $\mathrm{kDa}$ or higher, which have proven to have significant therapeutic value.

Mass spectra obtained using the HPLC interfaced on-line with Orbitrap MS for the $20 \mathrm{kDa}$ linear PEG and

Table 1. List of ISD fragment ions and their theoretical monoisotopic masses (Da) for the free peptide (see Figure 3 for experimental masses)

\begin{tabular}{lccccrrrrrr}
\hline & $\mathrm{R}^{*}$ & Ser & Gly & Gly & Val & Val & $\mathrm{K}^{*}$ & Lys & Asn & Phe \\
\hline \hline Ion & 12 & 13 & 14 & 15 & 16 & 17 & 18 & 19 & 20 & 21 \\
$\mathrm{c}$ & 1451.81 & & & & 1751.96 & 1851.02 & 2021.14 & 2149.24 & 2263.28 & 2410.35 \\
$\Delta$ & 0.10 & & & & 0.13 & 0.05 & 0.12 & 0.07 & 0.11 & 0.15 \\
$\mathrm{y}$ & & 1487.77 & 1657.88 & 1756.95 & 1856.02 & 1913.04 & 1970.06 & 2057.10 & 2214.18 & 2301.21 \\
$\Delta$ & & 0.14 & 0.12 & 0.13 & 0.12 & 0.13 & 0.12 & 0.14 & 0.14 & 0.15 \\
$\mathrm{z}+2$ & & 1472.76 & 1642.87 & 1741.94 & 1841.01 & 1898.03 & 1955.05 & 2042.09 & 2199.17 & 2286.20 \\
$\Delta$ & & 0.15 & 0.11 & 0.13 & 0.11 & 0.09 & 0.12 & 0.12 & 0.15 & 0.16 \\
& Asn & Ly & $\mathrm{K}^{*}$ & Val & Val & Gly & Gly & Ser & $R^{*}$ & Ser \\
\hline
\end{tabular}

$\Delta$ denotes the difference between the experimental and theoretical fragment ion mass ( $R^{*}$ for citrulline, $K^{*}$ for homoArg, and $X$ for naphthyl-Ala). 
the PEGylated peptide are shown in Figure 1a and b, respectively. Although attempts were made to obtain high-resolution mass spectral data in the Orbitrap, the size of the $20 \mathrm{kDa}$ PEG and the PEGylated peptide prevented it from traversing the C-trap, which is not uncommon for the analysis of molecules of $20 \mathrm{kDa}$ or greater. Therefore, the spectra shown in Figure 1 were actually acquired using the linear ion trap as a mass analyzer. The spectra are extremely complex and unresolved due to the heterogeneity associated with a large PEG, where the generation of multiply charged ions by ESI has further complicated the spectra. This is a typical observation for the ESI-MS analysis of PEGylated molecule, and interpretation of these spectra is essentially unachievable.

Given that singly charged ions are preferentially generated during the MALDI process, MALDI-TOF MS can be useful for the study of heterogeneous molecules. In addition, MALDI-MS is perhaps one of the most well suited techniques to analyze polymers and polymermodified molecules because of the simplicity of the mass spectra with hardly any unwanted fragmentation occurring in the polymer moieties during ionization. Figure 2 shows a representative mass spectrum from the PEGylated peptide using linear MALDI-TOF MS, where each of the oligomers can be clearly observed. The number average molecular weight, $\mathrm{Mn}$, of the PEGylated peptide was determined to be $24,575 \mathrm{Da}$ in this experiment. In contrast to $\mathrm{OH} / \mathrm{H}$-terminated PEGs, which are always observed as alkali-ionized species, the PEGylated peptide was observed in its protonated form in this experiment. However, only limited information pertaining to the peptide part of the PEGylated molecules can be obtained from MALDI-MS spectra alone. Furthermore, the MS/MS analysis using collisioninduced dissociation (CID) for ion activation, although generally possible for large molecules, is limited in this case, as the fragmentation will mainly occur on the PEG and provide little information on the peptide.

\section{Determination of PEGylation Site Using reISD}

PEGylation is generally carried out in a site-specific manner to produce well defined bioconjugates using numerous coupling strategies [7], and, therefore, it is important to monitor and confirm that PEGylation was successfully achieved at the desired locations. Previous studies have reported on the identification of the PEGylation site using enzymatic digestions. Although proteolytic digestion is a convenient means to study large biomolecules that are difficult to analyze in intact forms and often provides valuable information, it has a number of limitations as described earlier. In particular, as the molecule under the current study contains several non-natural amino acids that have no known enzymatic specificities, it would be difficult to correctly assign mass mapping data upon proteolysis. Ideally, determining the PEGylation site at the intact molecular level, or top-down analysis, can effectively prevent these problems and enable access to valuable information retained in the intact form.

Shown in Figure 3 is an enlarged reflector ISD spectrum obtained for the free peptide with $[\mathrm{M}+\mathrm{H}]^{+}$ of 3490.87 Da that was later PEGylated, where abundant signals for $\mathrm{c}-, \mathrm{y}-$, and $[\mathrm{z}+2]$-series of fragments, as also listed in Table 1 , were observed $[33,37]$. Complete sequence coverage of this model peptide was achieved based on ISD sequencing analysis and the homoArg [18], and the unmodified Lys ${ }^{19}$ residues were unequivocally assigned in the spectrum. Other fragmentation techniques such as CID or post-source decay (PSD) [47] using MALDI-MS often result in overwhelmingly degraded fragment products, including a-, d-, v, and $\mathrm{w}$-series, in addition to the more common $\mathrm{b}$ - and $y$-series of fragment ions [26, 38]. CID fails to provide reliable sequence readout at higher MW values, as the labile peptide bonds are predominantly dissociated. In contrast, ISD occurs uniformly across the sequence and provides relatively simple fragment ion spectra that generally include $c-$ and $(z+2)$-series of fragment ions, which result from the cleavage of $\mathrm{N}-\mathrm{C} \alpha$ bonds on the peptide backbone [39]. Although understanding of this fragmentation mechanism is not complete, it has been proposed that the process follows chemical activation by hydrogen radical transfer reactions in the MALDI plume. As a limitation, however, ISD-based MALDITOF MS is a pseudo-MS/MS technique with in-source fragmentation being incompatible with precursor ion selection. This requires that the sample is highly pure or

Table 1. Continued

\begin{tabular}{ccccccrrrr}
\hline Val & Pro & Thr & Asp & Val & Gly & Pro & X & Ala & Phe \\
\hline \hline 22 & 23 & 24 & 25 & 26 & 27 & 28 & 29 & 30 & 31 \\
& & & 2822.54 & 2921.61 & & 3075.69 & & & \\
2414.30 & 2527.38 & 2584.40 & 2655.44 & 2768.52 & 2925.61 & 3062.67 & 3163.72 & 3262.78 & 3490.87 \\
0.16 & 0.14 & 0.15 & 0.13 & 0.14 & 0.15 & 0.09 & 0.06 & 0.13 & 0.11 \\
2399.29 & 2512.37 & 2569.39 & 2640.43 & 2753.51 & 2910.60 & 3047.66 & 3148.71 & 3247.77 \\
0.17 & 0.11 & 0.18 & 0.16 & 0.18 & 0.17 & 0.17 & 0.17 & 0.14 & Thr \\
Leu & Leu & Gly & Ala & Leu & $R^{*}$ & His & Thr & Tral \\
\hline
\end{tabular}


ISD-MALDI MS to be combined with off-line chromatographic separation.

Figure 4 shows an ISD spectrum obtained for the protonated PEGylated peptide that uses the same free peptide as above for conjugation. In the resulting fragment ion spectrum, all fragment ions were observed in the protonated forms, $\mathrm{c}$ - and $\mathrm{y}$-series, as summarized in Table 2; they clearly show a fragmentation pattern comparable to that of the protonated free peptide. However, the ion series for the PEGylated peptide were observed to be truncated at $\operatorname{Lys}^{19}$ i.e., at $\mathrm{c}_{18}$ and $\mathrm{y}_{12}$, respectively. This particular truncation of sequence readout serves as a strong indication for the presence of a modification at $\mathrm{Lys}^{19}$ here as the PEGylation site, in which the PEG moiety of ca. $20 \mathrm{kDa}$ is too large to be monitored in the reISD spectra of the PEGylated peptide. Based on high mass accuracy, as shown in Tables 1 and 2, and peak resolution in the range of 10,000 to 20,000 for reISD fragment peaks, this technique is robust enough to detect, localize, and assign modifications of very small molecular mass differences, such as deamidation.

Previously, it has been demonstrated that fragment ion series using top-down ISD are truncated at the position of cyclization, as in proline disulfide bonds [33, 36], and heme-crosslinks in cytochrome $c$ [34]. Cyclization and large modifications introduced a gap or a truncation in sequencing analysis, and, therefore, helped predict the presence of such modifications. In this experiment, PEG being a large and heterogeneous molecule, MS sequencing information on Lys ${ }^{19}$ is missing as a result of PEG attachments. As fragments for the modified side chains of proteins, such as phosphates, glycans, or disulfide bonds, are typically not observed in the spectra from ISD, ECD, and ETD, in contrast to
CID [30], the truncation of the ion series at Lys $^{19}$ is in line with the prediction that a conjugation with a $20 \mathrm{kDa}$ PEG would truncate the sequence readout at that particular position. It is believed that our observation resembles the case of disulfide bonds characterization or the study of proline containing sequences, where their presence is characterized by missing sequencing information due to the presence of cyclic moiety [32]. Recently, reductive treatment of the disulfides in proteins by the use of reductive MALDI matrices [48] has shown to remove the cyclic structures and enable the direct sequence readout from the linearized protein.

The model compound studied in this preliminary study may represent a relatively simple form of a PEGylated molecule suitable for a straightforward analysis. It basically contains a single potential PEGylation site, as the N-terminus of the peptide is acetylated and not susceptible to the current PEGylation chemistry. However, when the compounds to be studied have added complexity in this regard, further study may become necessary to validate the ability of the described approach to distinguish different PEGylation sites and quantify the respective degree of modification [48]. Nevertheless, the ISD-based approach presented in this work has proven to be capable of rapidly suggesting the PEGylation site of a PEGylated biomolecule by requiring minimal sample preparation.

\section{Conclusions}

The ISD-based MALDI MS technique has already successfully shown its usefulness for the structural study of intact proteins and biologically occurring modifications due to its unique ion fragmentation characteristics. The work described herein suggests that applications of the

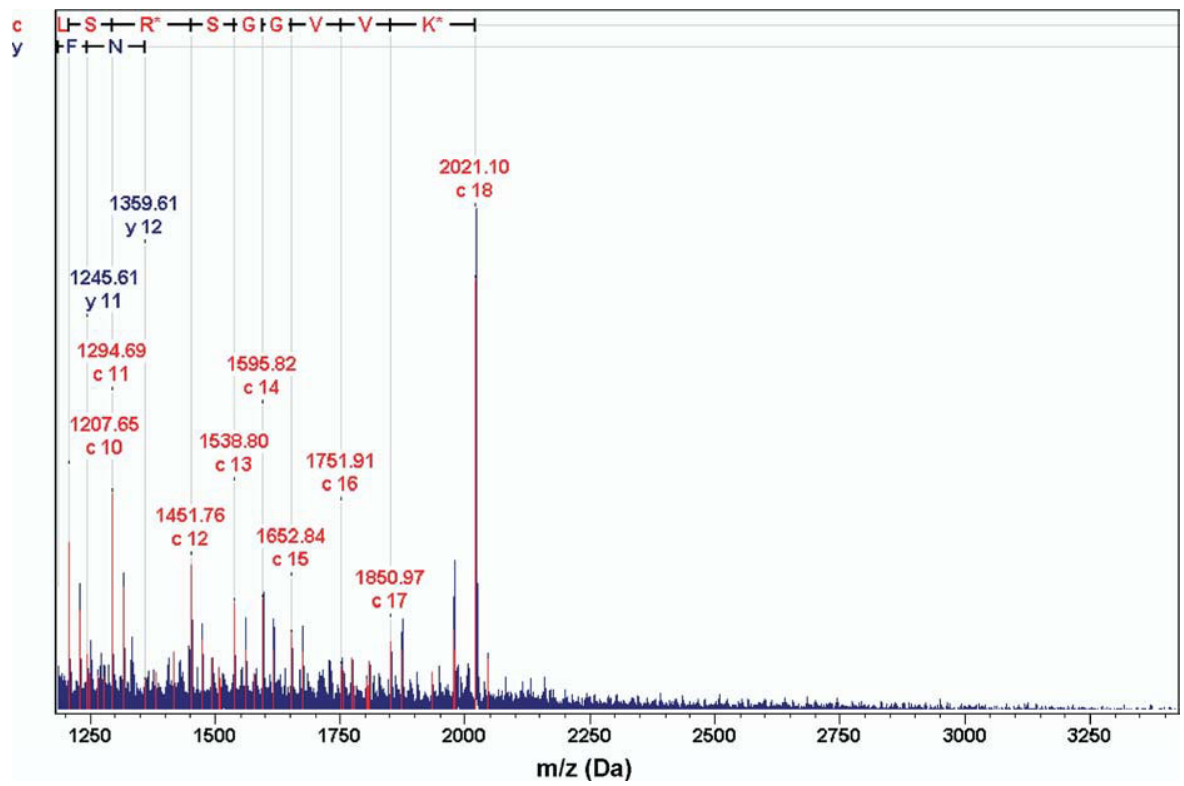

Figure 4. reISD-MALDI MS spectrum obtained for the PEGylated peptide ( $R^{*}$ for citrulline; $K^{*}$ for homoArg). Matching fragment ions are assigned. 


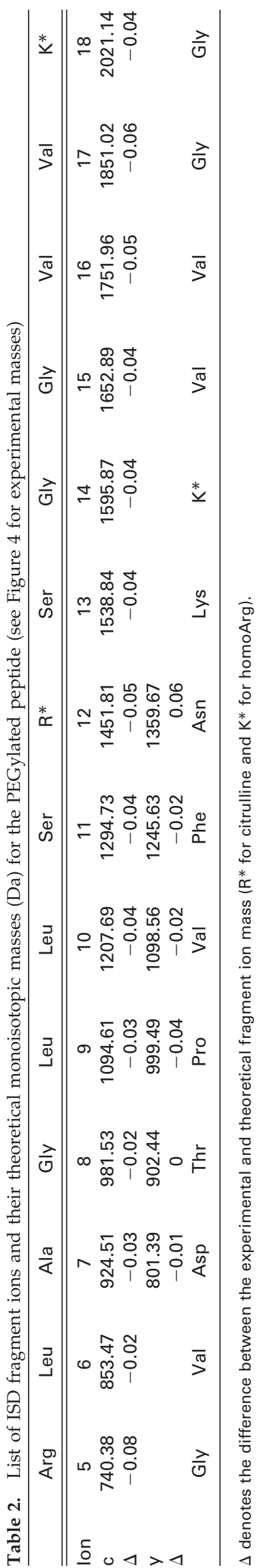

ISD can also be extended to study structural aspects of PEGylated biotherapeutics. MALDI-MS is to play a significant role in the development of polymers with pharmaceutical significance due to its advantages described earlier. MALDI-MS has a great potential to rapidly provide very useful structural information from heterogeneous molecules with clearly distinguishable fragmentation properties of the different chemical moieties. It is also important to emphasize that the entire analysis was performed at the intact molecular level without altering the original model compound, and, therefore, dramatically improved throughput and simplified data interpretation was achieved. Uniquely important for this application was the fact that no precursor ion selection was necessary in reISD, which is not possible with typical MS/MS experiments for molecules exhibiting a high degree of polymerization at high MW. ReISD spectra obtained in this work clearly provided detailed information about the sequence and suggested the conjugation status of individual amino acid residues in the PEGylated peptide.

\section{Acknowledgments}

The authors thank Dr. Ryan Holder of Peptide Research and Discovery and Dr. Colin Gegg and Marie Wright of Protein Science at Amgen, Inc. for generously providing samples used in this study. The authors are also grateful to Dr. David Semin and Dr. Paul Schnier for critical review and discussion during the preparation of the manuscript.

\section{References}

1. Veronese, F. M.; Pasut, G. PEGylation, Successful Approach to Drug Delivery. Drug Discov. Today. 2005, 10, 1451-1458.

2. Roberts, M. J.; Bentley, M.D.; Harris, J. M. Chemistry for Peptide and Protein PEGylation. Adv. Drug Delivery Rev. 2002, 54, 459-476.

3. Harris, J. M.; Chess, R. B. Effect of PEGylation on Pharmaceuticals. Nat. Rev. Drug Discov. 2003, 2, 214-221.

4. Greenwald, R. B.; Choe, Y. H.; McGuire, J.; Conover, C. D. Effective Drug Delivery by PEGylated Drug Conjugates. Adv. Drug Delivery Rev. 2003, 55, 217-250.

5. Molineux, G. Pegylation: Engineering Improved Biopharmaceuticals for Oncology. Pharmacotherapy 2003, 23, 3S-8S

6. Yang, Z.; Wang, J.; Lu, Q.; Xu, J.; Kobayashi, Y.; Takakura, T.; Takimoto, A.; Yoshioka, T.; Lian, C.; Chen, C.; Zhang, D.; Zhang, Y.; Li, S.; Sun, X.; Tan, Y.; Yagi, S.; Frenkel, E. P.; Hoffman, R. M. PEGylation Confers Greatly Extended Half-Life and Attenuated Immunogenicity to Recombinant Methioninase in Primates. Cancer Res. 2004, 64, 6673-6678.

7. Kinstler, O.; Molineux, G.; Treuheit, M.; Ladd, D.; Gegg, C. Mono-NTerminal Poly(Ethylene Glycol)-Protein Conjugates. Adv. Drug Delivery Rev. 2002, 54, 477-485.

8. Rajan, R. S.; Li, T.; Aras, M.; Sloey, C.; Sutherland, W.; Arai, H.; Briddell, R.; Kinstler, O.; Lueras, A. M. K.; Zhang, Y.; Yeghnazar, H.; Treuheit, M.; Brems, D. N. Modulation of Protein Aggregation by Polyethylene Glycol Conjugation: GCSF as a Case Study. Protein Sci. 2006, 15, 1063-1075.

9. Ramon, J.; Saez, V.; Baez, R.; Aldana, R.; Hardy, E. PEGylated interferonz2b: A Branched $40 \mathrm{~K}$ Polyethylene Glycol Derivative. Pharmaceut. Res. 2005, 22, 1374-1386.

10. DeNardo, S. J.; Yao, Z.; Lam, K. S.; Lamborn, K. R.; O-Donnell, R. T. Denardo, G. L. Effect of Molecular Size of Pegylated Peptide on the Pharmacokinetics and Tumor Targeting in Lymphoma-Bearing Mice. Clin. Cancer Res. 2003, 9, 3854s-3864s.

11. Bagal, D.; Zhang, H.; Schnier, P. D. Gas-Phase Proton-Transfer Chemistry Coupled with TOF Mass Spectrometry and Ion Mobility-MS for the Facile Analysis of Poly(Ethylene Glycols) and PEGylated Polypeptide Conjugates. Anal. Chem. 2008, 80, 2408-2418.

12. Vestling, M. M.; Murphy, C. M.; Keller, D. A.; Fenselau, C.; Dedinas, J.; Ladd, D. L.; Olsen, M. A. A Strategy for Characterization of Polyethylene Glycol-Derivatized Superoxide Dismutase. Drug Metab. Dispos. 1993, 21, 911-917. 
13. Iafelice, R.; Cristoni, S.; Caccia, D.; Russo, R.; Rossi-Bernardi, L.; Lowe, K. C.; Perrella, M. Identification of the Sites of Deoxyhemoglobin PEGylation. Biochem. J. 2007, 403, 189-196.

14. Stigsnaes, P.; Frokjaer, S.; Bjerregaard, S.; van de Weert, M.; Kingshott, P.; Moeller, E. H. Characterization and Physical Stability of PEGylated Glucagon. Int. J. Pharm. 2007, 330, 89-98.

15. Roberts, M. J.; Harris, J. M. Attachment of Degradable Poly(Ethylene Glycol) to Proteins Has the potential to Increase a Therapeutic Efficacy. J. Pharm. Sci. 1998, 87, 1440-1445.

16. Karas, M.; Hillenkamp, F. Laser Desorption Ionization of Proteins with Molecular Masses Exceeding 10,000 Daltons. Anal. Chem. 1988, 60, $2299-2301$.

17. Tanaka, K.; Wak, H.; Ido, Y.; Akita, S.; Yoshida, Y.; Yoshida, T. Protein and Polymer Analyses up to $\mathrm{m} / \mathrm{z} 100000$ by Laser Ionization Time-ofFlight Mass Spectrometry. Rapid Commun. Mass Spectrom. 1988, 2, 151-153.

18. Karas, M.; Gluckmann, M.; Schafer, J. Ionization in Matrix-Assisted Laser Desorption/Ionization: Singly Charged Molecular Ions are the lucky Survivors. J. Mass Spectrom. 2000, 35, 1-12.

19. Giannakopulos, A. E.; Thomas, N.; Colburn, A. W.; Reynolds, D. J.; Raptakis, E. N.; Makarov, A. A.; Derrick, P. J. Tandem Time-of-Flight Mass Spectrometer (TOF-TOF) with a Quadratic-Field Ion Mirror. Rev. Sci. Instrum. 2002, 73, 2115-2123.

20. Schriemer, D. C.; Li, L. Detection of High Molecular Weight Narrow Polydisperse Polymers up to 1.5 Million Daltons by MALDI Mass Spectrometry. Anal. Chem. 1996, 68, 2721-2725.

21. Schriemer, D. C.; Li, L. Mass Discrimination in the Analysis of Polydisperse Polymers by MALDI Time-of-Flight Mass Spectrometry. 1. Sample Preparation and Desorption/Ionization Issues. Anal. Chem. 1997, 69, 4169-4175.

22. Schriemer, D. C.; Li, L. Mass Discrimination in the Analysis of Polydisperse Polymers by MALDI Time-of-Flight Mass Spectrometry. 2. Instrumental Issues. Anal. Chem. 1997, 69, 4176-4183.

23. Nielen, M. W. F. MALDI Time-of-Flight Mass Spectrometry of Synthetic Polymers. Mass Spectrom. Rev. 1999, 18, 309-344.

24. Cotter, R. J.; Griffith, W.; Jelinek, C. Tandem Time-of-Flight (TOF/TOF) Mass Spectrometry and the Curve-Field Reflectron. J. Chromatogr. B. 2007, 855, 2-13.

25. Suckau, D.; Resemann, A.; Schuerenberg, M.; Hufnagel, P.; Franzen, J. Holle, A. A novel MALDI LIFT-TOF/TOF Mass Spectrometer for Proteomics. Anal. Bioanal. Chem. 2003, 376, 952-965.

26. Macht, M.; Asperger, A.; Deininger, S.-O. Comparison of Laser-Induced Dissociation and High-Energy Collision-Induced Dissociation Using Matrix-Assisted Laser Desorption/Ionization Tandem Time-of-Flight (MALDI-TOF/TOF) for Peptide and Protein Identification. Rapid Commun. Mass Spectrom. 2004, 18, 2093-2105.

27. Yoo, C.: Zhao, J.: Pal, M.; Hersberger, K. Huber, C. G.; Simeone, D. M. Beer, D. G.; Lubman, D. M. Automated Integration of Monolith-Based Protein Separation with On-Plate Digestion for Mass Spectrometric Analysis of Esophageal Adenocarcinoma Human Epithelial Samples. Electrophoresis 2006, 27, 3643-3451.

28. Demirev, P. A.; Feldman, A. B.; Kowalski, P.; Lin, J. S. Top-Down Proteomics for Rapid Identification of Intact Microorganisms. Anal. Chem. 2005, 77, 7455-7461.

29. Liu, Z.; Schey, K. Optimization of a MALDI-TOF/TOF Mass Spectrometer for Intact Protein Analysis. J. Am. Soc. Mass Spectrom. 2005, 16, $482-490$.

30. Samyn, B.; Debyser, G.; Sergeant, K.; Devreese, B.; Beeumen, J. V. A Case Study of de Novo Sequence Analysis of N-Sulfonated Peptides by
MALDI-TOF/TOF Mass Spectrometry. J. Am. Soc. Mass Spectrom. 2004, $15,1838-1852$.

31. Hardouin, J. Protein Sequence Information by Matrix-Assisted Laser Desorption/Ionization In-Source Decay Mass Spectrometry. Mass Spectrom. Rev. 2007, 26, 672-682.

32. Hardouin, J.; Hubert-Roux, M.; Delmas, A. F.; Lange, C. Identification of Isoenzymes Using Matrix-Assisted Laser Desorption/Ionization Timeof-Flight Mass Spectrometry. Rapid Commun. Mass Spectrom. 2006, 20, 725-732.

33. Suckau, D.; Resemann, A. T3-Sequencing: Targeted Characterization of the N- and C-Termini of Undigested Proteins by Mass Spectrometry. Anal. Chem. 2003, 75, 5817-5824.

34. Lennon, J. J.; Walsh, K. A. Direct Sequence Analysis of Proteins by In-Source Fragmentation During Delayed Ion Extraction. Protein Sci. 1997, 6, 2446-2453.

35. Brown, R. S.; Lennon, J. J. Sequence-Specific Fragmentation of MatrixAssisted Laser-Desorbed Protein/Peptide Ions. Anal. Chem. 1995, 67, 3990-3999.

36. Schnaible, V.; Wefing, S.; Resemann, A.; Suckau, D.; Bucker, A.; WolfKummeth, S.; Hoffmann, D. Screening for Disulfide Bonds in Proteins by MALDI In-Source Decay and LIFT-TOF/TOF MS. Anal. Chem. 2002, $74,4980-4988$

37. Demeure, K.; Quinton, L.; Gabelica, V.; Pauw, E. D. Rational Selection of the Optimum MALDI Matrix for Top-Down Proteomics by In-Source Decay. Anal. Chem. 2007, 79, 8678-8685.

38. Takayama, M. In-Source Decay Characteristics of Peptides in MatrixAssisted Laser Desorption/Ionization Time-of-Flight Mass Spectrometry. J. Am. Soc. Mass Spectrom. 2001, 12, 420-427.

39. Kocher, T.; Engstrom, A.; Zubarev, R. A. Fragmentation of Peptides in MALDI In-Source Decay Mediated by Hydrogen Radicals. Anal. Chem. 2005, 77, 172-177.

40. Gegg, C.; Miranda, L.; Walker, K.; Johnson, E.; Holder, J. R.; Wright, M. E.; D'Amico, D. C. WO 2007/048026.

41. Holle, A.; Haase, A.; Kayser, M.; Hohndorf, J. Optimizing UV Laser Focus Profiles for Improved MALDI Performance. J. Mass Spectrom. 2006, 41, 705-716.

42. Na, D. H. Lee, K. C. Capillary Electrophoretic Characterization of PEGylated Human Parathyroid Hormone with Matrix-Assisted Laser Desorption/Ionization Time-of-Flight Mass spectrometry. Anal. Biochem. 2004, 331, 322-328.

43. Na, D. H.; Park, M. O.; Choi, S. Y.; Kim, Y. S.; Lee, S. S.; Yoo, S. D.; Lee H. S.; Lee, K. C. Identification of the Modifying Sites of MonoPEGylated Salmon Calcitonins by Capillary Electrophoresis and MALDI-TOF Mass Spectrometry. J. Chromatogr. B. 2001, 754, 259-263.

44. Dou, H.; Zhang, M.; Zhang, Y.; Yin, C. Synthesis and Purification of mono-PEGylated insulin. Chem. Biol. Drug Design. 2007, 69, 132-138.

45. Veronese, F. M. Peptide and Protein PEGylation: A Review of Problems and Solution. Biomaterials 2001, 22, 405-417.

46. Esposito, P.; Barbero, L.; Caccia, P.; D'Antonio, M.; Pizuet, G.; Veronese F. M. PEGylation of Growth Hormone-Releasing Hormone (GRF) Analogues. Adv. Drug Delivery Rev. 2003, 55, 1279-1291.

47. Kaufmann, R.; Kirsch, D.; Spengler, B. Sequencing of Peptides in a Time-of-Flight Mass Spectrometer: Evaluation of Postsource Decay Following Matrix-Assisted Laser Desorption Ionization (MALDI). Int. J. Mass. Spectrom. Ion Processes 1994, 131, 355-385.

48. Quinton, L.; Demeure, K.; Dobson, R.; Gilles, N.; Gabelica, V.; Pauw, E. D. New Method for Characterizing Highly Disulfide-Bridged Peptides in Complex Mixtures: Application to Toxin Identification from Crude Venoms. J. Proteome Res. 2007, 6, 3216-3223. 